Research Article

\title{
Characteristics of Regular Functions Defined on a Semicommutative Subalgebra of 4-Dimensional Complex Matrix Algebra
}

\author{
Ji Eun Kim (1D) \\ Department of Mathematics, Dongguk University, Gyeongju-Si 38066, Republic of Korea \\ Correspondence should be addressed to Ji Eun Kim; jeunkim@pusan.ac.kr
}

Received 30 July 2021; Accepted 20 November 2021; Published 10 December 2021

Academic Editor: V. Ravichandran

Copyright (C) 2021 Ji Eun Kim. This is an open access article distributed under the Creative Commons Attribution License, which permits unrestricted use, distribution, and reproduction in any medium, provided the original work is properly cited.

\begin{abstract}
In this paper, we give an extended quaternion as a matrix form involving complex components. We introduce a semicommutative subalgebra $\mathbb{C}\left(\mathbb{C}^{2}\right)$ of the complex matrix algebra $M(4, \mathbb{C})$. We exhibit regular functions defined on a domain in $\mathbb{C}^{4}$ but taking values in $\mathbb{C}\left(\mathbb{C}^{2}\right)$. By using the characteristics of these regular functions, we propose the corresponding Cauchy-Riemann equations. In addition, we demonstrate several properties of these regular functions using these novel Cauchy-Riemann equations. Mathematical Subject Classification is 32G35, 32W50, 32A99, and 11E88.
\end{abstract}

\section{Introduction}

Introduced by Hamilton in 1894, quaternions form an algebra generated as a noncommutative division (associative) algebra. Quaternions are used in physics and engineering fields such as electromechanics, quantum mechanics, and $3 \mathrm{D}$ animation (see [1-3]). To increase the utilization of quaternions and expand their application, several researchers have attempted to modify, supplement, or expand quaternions. In particular, considering that quaternions form a number system extending the complex numbers, there have been several studies already made since the past few decades regarding holomorphic functions of a quaternion variable. These researchers have investigated whether the properties of complex functions and the definition of holomorphic functions are applicable to functions of a quaternion variable. To do this, researchers have defined holomorphic (regular) functions of a quaternion variable and they have investigated their properties. Fueter [4] defined regular functions over the quaternion field identified with $\mathbb{R}^{4}$. Based on this definition, Fueter investigated a generalization of the Cauchy-Riemann equations in the complex holomorphic function theory. Delanghe [5] established Stokes' theorem and Cauchy's and Green's formulas for functions with values in Clifford algebra over a quadratic $n$-dimensional real vector space $V$ with an orthogonal base. Using a generalization of the Cauchy-Riemann equation, Ryan [6] introduced a regularity of quaternion-valued functions and developed a regular function theory of complex Clifford algebra. Sudbery [7] demonstrated basic algebraic properties of quaternions and quaternionic differential forms. Using exterior differential calculus, Sudbery proposed new and simple proofs of most of the main theorems and clarified the relationship between quaternionic analysis and complex analysis. Naser [8] and Nôno [9] introduced certain quaternionic differential operators and defined the hyperholomorphy of quaternionic functions. Nôno and Inenaga [10] developed hyperholomorphic functions of quaternionic variables as holomorphic function theory of $\mathbb{C}^{2}$. Kim et al. [11, 12] researched the properties of regular functions with values in the three-dimensional real skew field (called ternary field $\mathbb{T}$ ) and reduced quaternions using Clifford analysis. They proposed the corresponding Cauchy-Riemann equations with applications defined on $\mathbb{T}$. Sommen [13] constructed kernels and monogenic and holomorphic functions, leading to connections between the theory of holomorphic functions of several variables and the theory of monogenic functions. 
Based on these studies, in this paper, we propose a form distinct from the previously attempted expansion of quaternions. In previous studies, extensions of the quaternionic number system and various combinations of quaternions were suggested. Baez [14] applied octonions to spinors, Bott periodicity, projective and Lorentzian geometry, Jordan algebras, and exceptional Lie groups. Baez also dealt with their applications in quantum logic, special relativity, and supersymmetry. Imaeda $\mathrm{K}$ and Imaeda $\mathrm{M}$ [15] presented a 16-dimensional Cayley-Dickson algebra and found its algebraic properties, zero-divisors, and solutions to a general linear equation. Tian [16] provided a complete investigation of real matrix representations of octonions and considered their various applications to octonions, based on the fact that the octonion is an extension of the quaternion by the Cayley-Dickson construction. Gotô and Nôno [17] established a commutative algebra $\mathbb{C}\left(\mathbb{C}^{2}\right)$ identified with $\mathbb{C}$ as the subalgebra of the four-dimensional real matrix algebra $M(4, \mathbb{R})$. They provided a regularity and properties of the functions of two complex variables with values in $\mathbb{C}\left(\mathbb{C}^{2}\right)$. Rudin [18] studied the integral formulas of the holomorphic functions of several complex variables and presented a function theory of including the boundary behavior of complex functions, complex-tangential phenomena, and quantitative theorems about zero-varieties in the unit ball of $\mathbb{C}^{n}$.

In this paper, we give algebra using the matrix as the basis by mapping the basis of the quaternion to matrix form. We define an algebraic system based on a matrix with a matrix as a component. This can be expressed as a matrix with complex numbers as components while retaining the characteristics of the basis for generating quaternions. Furthermore, a $4 \times 4$ matrix is constructed with a matrix that is isomorphic to the basis of quaternions. The algebra introduced in this paper has eight bases, some of which are commutative for products and some are noncommutative. Therefore, in this paper, a function is defined on an algebra generated by a semicommutative basis. Regularity of functions and properties of these regular functions is investigated. In addition, the corresponding Cauchy-Riemann equations, which can be an alternative of the definition of holomorphic function in complex analysis, are derived for the algebra.

We develop the foundation of a function theory over a subalgebra of four-dimensional matrix algebra to replace the basis of the quaternion. In Section 2, we present preliminaries and notations required for the theory presented in this paper. Sections 3 and 4 present the definitions and propositions related to the semicommutative subalgebra $\mathbb{C}\left(\mathbb{C}^{2}\right)$ of the complex matrix algebra $M(4, \mathbb{C})$, and we provide a regularity of functions defined on a domain in $\mathbb{C}^{4}$ with values in $\mathbb{C}\left(\mathbb{C}^{2}\right)$. From the notion of regularity over $\mathbb{C}\left(\mathbb{C}^{2}\right)$, we propose corresponding Cauchy-Riemann equations and several properties of regular functions in $\mathbb{C}\left(\mathbb{C}^{2}\right)$. Finally, in Section 5, the conclusions of this paper are presented.

\section{Preliminaries and Notations}

In this section, we give notations that are needed to prove our main results. Let $M(n, \mathbb{F})$ be the ring of all $n \times n$ matrices over the field $\mathbb{F}$. For our purposes, we would be interested in the case where $n=2$ and $\mathbb{F}=\mathbb{C}$. Put

$$
\begin{aligned}
& e_{0}=\left(\begin{array}{ll}
1 & 0 \\
0 & 1
\end{array}\right), \\
& e_{1}=\left(\begin{array}{cc}
i & 0 \\
0 & -i
\end{array}\right), \\
& e_{2}=\left(\begin{array}{cc}
0 & 1 \\
-1 & 0
\end{array}\right), \\
& e_{3}=\left(\begin{array}{ll}
0 & i \\
i & 0
\end{array}\right) .
\end{aligned}
$$

These have the following rules:

$$
\begin{aligned}
e_{0}^{2} & =e_{0}, \\
e_{1}^{2} & =e_{2}^{2}=e_{3}^{2}=-e_{0}, \\
e_{1} e_{2} & =e_{3}=-e_{2} e_{1}, \\
e_{2} e_{3} & =e_{1}=-e_{3} e_{2}, \\
e_{3} e_{1} & =e_{2}=-e_{1} e_{3} .
\end{aligned}
$$

Let $\mathcal{S}$ be a set of matrices defined as follows:

$$
\mathcal{S}=\left\{\sum_{r=0}^{3} x_{r} e_{r} \mid x_{r} \in \mathbb{R}(r=0,1,2,3)\right\} .
$$

From the rules of $e_{r}(r=0,1,2,3)$, we note that $\mathcal{S}$ is a noncommutative subalgebra of $M(2, \mathbb{C})$. By using the matrices $e_{r}(r=0,1,2,3)$, we define four $4 \times 4$ matrices: 


$$
\begin{aligned}
& \varepsilon_{0}=\left(\begin{array}{ll}
e_{0} & \mathbf{0} \\
\mathbf{0} & e_{0}
\end{array}\right), \\
& \varepsilon_{1}=\left(\begin{array}{cc}
e_{1} & 0 \\
\mathbf{0} & -e_{1}
\end{array}\right), \\
& \varepsilon_{2}=\left(\begin{array}{cc}
e_{0} & \mathbf{0} \\
\mathbf{0} & -e_{0}
\end{array}\right), \\
& \varepsilon_{3}=\left(\begin{array}{cc}
e_{1} & \mathbf{0} \\
\mathbf{0} & e_{1}
\end{array}\right), \\
& \varepsilon_{4}=\left(\begin{array}{cc}
0 & e_{2} \\
-e_{2} & 0
\end{array}\right), \\
& \varepsilon_{5}=\left(\begin{array}{cc}
0 & e_{3} \\
e_{3} & 0
\end{array}\right), \\
& \varepsilon_{6}=\left(\begin{array}{cc}
0 & e_{2} \\
e_{2} & 0
\end{array}\right), \\
& \varepsilon_{7}=\left(\begin{array}{cc}
0 & e_{3} \\
-e_{3} & 0
\end{array}\right),
\end{aligned}
$$

where 0 is the $2 \times 2$ null matrix. Then, we have the following properties:

$$
\begin{aligned}
& \varepsilon_{0}^{2}=\varepsilon_{2}^{2}=\varepsilon_{4}^{2}=\varepsilon_{6}^{2}=\varepsilon_{0}, \\
& \varepsilon_{1}^{2}=\varepsilon_{3}^{2}=\varepsilon_{5}^{2}=\varepsilon_{7}^{2}=-\varepsilon_{0},
\end{aligned}
$$

and

$$
\begin{aligned}
& \varepsilon_{1} \varepsilon_{2}=\varepsilon_{3}=\varepsilon_{2} \varepsilon_{1}, \\
& \varepsilon_{1} \varepsilon_{6}=\varepsilon_{7}=\varepsilon_{6} \varepsilon_{1}, \\
& \varepsilon_{5} \varepsilon_{4}=\varepsilon_{1}=\varepsilon_{4} \varepsilon_{5}, \\
& \varepsilon_{2} \varepsilon_{5}=\varepsilon_{7}=-\varepsilon_{5} \varepsilon_{2}, \\
& \varepsilon_{5} \varepsilon_{3}=\varepsilon_{6}=-\varepsilon_{3} \varepsilon_{5}, \\
& \varepsilon_{3} \varepsilon_{4}=\varepsilon_{7}=-\varepsilon_{4} \varepsilon_{3}, \\
& \varepsilon_{6} \varepsilon_{4}=\varepsilon_{2}=-\varepsilon_{4} \varepsilon_{6} .
\end{aligned}
$$

Now, let the set $\mathcal{S}(4, \mathbb{C})$ be a subalgebra of $M(4, \mathbb{C})$, written as

$$
\mathcal{S}(4, \mathbb{C})=\left\{z=\sum_{r=0}^{7} x_{r} \varepsilon_{r} \mid x_{r} \in \mathbb{R}, r=0,1, \ldots, 7\right\},
$$

Here, we deal with $\varepsilon_{0}$ as 1 . The element $z$ of $\mathcal{S}(4, \mathbb{C})$ is also written as

$$
\begin{aligned}
z= & \left(x_{0}+x_{1} \varepsilon_{1}+x_{2} \varepsilon_{2}+x_{3} \varepsilon_{3}\right)+\left(x_{4}+x_{5} \varepsilon_{1}+x_{6} \varepsilon_{2}+x_{7} \varepsilon_{3}\right) \varepsilon_{4} \\
= & \left\{\left(x_{0}+x_{1} \varepsilon_{1}\right)+\left(x_{2}+x_{3} \varepsilon_{1}\right) \varepsilon_{2}\right\} \\
& +\left\{\left(x_{4}+x_{5} \varepsilon_{1}\right)+\left(x_{6}+x_{7} \varepsilon_{1}\right) \varepsilon_{2}\right\} \varepsilon_{4} .
\end{aligned}
$$

From the above expressions, by simply making the following substitutions, we put

$$
\begin{aligned}
& \zeta_{0}=x_{0}+x_{1} \varepsilon_{1}, \\
& \zeta_{1}=x_{2}+x_{3} \varepsilon_{1}, \\
& \zeta_{2}=x_{4}+x_{5} \varepsilon_{1}, \\
& \zeta_{3}=x_{6}+x_{7} \varepsilon_{1},
\end{aligned}
$$

and we obtain

$$
\begin{aligned}
& z_{0}=\zeta_{0}+\zeta_{1} \varepsilon_{2}, \\
& z_{1}=\zeta_{2}+\zeta_{3} \varepsilon_{2}, \\
& \bar{z}_{0}=\bar{\zeta}_{0}+\bar{\zeta}_{1} \varepsilon_{2}, \\
& \bar{z}_{1}=\bar{\zeta}_{2}+\bar{\zeta}_{3} \varepsilon_{2}, \\
& \widetilde{z}_{0}=\zeta_{0}-\zeta_{1} \varepsilon_{2}, \\
& \widetilde{z}_{1}=\zeta_{2}-\zeta_{3} \varepsilon_{2},
\end{aligned}
$$

where $\bar{z}_{t}(t=0,1)$ denotes the complex conjugate of the complex components of $\varepsilon_{1}$ and $\widetilde{z}_{t}$ is a changing symbol of a part of the basis of $\varepsilon_{2}$. Then, the elements of $\mathcal{S}(4, \mathbb{C})$ can be represented in the form $z_{0}+z_{1} \varepsilon_{4}$. Let $\mathbb{C}\left(\mathbb{C}^{2}\right)$ be the set $\mathcal{S}(4, \mathbb{C})$, which is the set of elements of $\mathcal{S}(4, \mathbb{C})$ expressed in the form of $z_{0}+z_{1} \varepsilon_{4}$, denoted by

$$
\mathbb{C}\left(\mathbb{C}^{2}\right)=\left\{z=z_{0}+z_{1} \varepsilon_{4} \mid z_{0}, z_{1} \in M(4, \mathbb{C})\right\} .
$$

In addition, the conjugate of $z$ in $\mathbb{C}\left(\mathbb{C}^{2}\right)$ is defined as $z=\bar{z}_{0}+\bar{z}_{1} \varepsilon_{4}$. Explicitly, we have

$$
\begin{aligned}
\bar{z} & =x_{0}-x_{1} \varepsilon_{1}+x_{2} \varepsilon_{2}-x_{3} \varepsilon_{3}+x_{4} \varepsilon_{4}-x_{5} \varepsilon_{5}+x_{6} \varepsilon_{6}-x_{7} \varepsilon_{7} \\
& =\left(x_{0}-x_{1} \varepsilon_{1}+x_{2} \varepsilon_{2}-x_{3} \varepsilon_{3}\right)+\left(x_{4}-x_{5} \varepsilon_{1}+x_{6} \varepsilon_{2}-x_{7} \varepsilon_{3}\right) \varepsilon_{4} \\
& =\left\{\left(x_{0}-x_{1} \varepsilon_{1}\right)+\left(x_{2}-x_{3} \varepsilon_{1}\right) \varepsilon_{2}\right\}+\left\{\left(x_{4}-x_{5} \varepsilon_{1}\right)+\left(x_{6}-x_{7} \varepsilon_{1}\right) \varepsilon_{2}\right\} \varepsilon_{4} \\
& =\left(\bar{\zeta}_{0}+\bar{\zeta}_{1} \varepsilon_{2}\right)+\left(\bar{\zeta}_{2}+\bar{\zeta}_{3} \varepsilon_{2}\right) \varepsilon_{4} \\
& =\bar{z}_{0}+\bar{z}_{1} \varepsilon_{4} .
\end{aligned}
$$

For $z, w \in \mathbb{C}\left(\mathbb{C}^{2}\right)$, we can write $z=z_{0}+z_{1} \varepsilon_{4}$ and $w=w_{0}+w_{1} \varepsilon_{4}$, then owing to the identities

$$
\begin{aligned}
& z_{0} \varepsilon_{4}=\varepsilon_{4} \widetilde{z}_{0}, \\
& z_{1} \varepsilon_{4}=\varepsilon_{4} \widetilde{z}_{1} .
\end{aligned}
$$

Thus, we obtain

$$
z w=\left(z_{0} w_{0}+z_{1} \widetilde{w}_{1}\right)+\left(z_{0} w_{1}+z_{1} \widetilde{w}_{0}\right) \varepsilon_{4} .
$$

Since $\varepsilon_{1} \varepsilon_{2}=\varepsilon_{2} \varepsilon_{1}$, we have

$$
z_{r} w_{t}=w_{t} z_{r}, \quad(r, t=0,1) .
$$

However,

$$
\begin{aligned}
& z_{r} \varepsilon_{4}=\varepsilon_{4} \widetilde{z}_{r}, \\
& w_{t} \varepsilon_{4}=\varepsilon_{4} \widetilde{w}_{t} .
\end{aligned}
$$

Although, as a result, $z w \neq w z$, it is equal to a part of $z w$ and $w z$; hence, $\mathbb{C}\left(\mathbb{C}^{2}\right)$ is a semicommutative subalgebra of $M(4, \mathbb{C})$. We first obtain an expression for $z^{*}$ in order to define $\|z\|$. Let $z^{*}=\overline{\left(z^{t}\right)}$, where $z^{t}$ is the transposed matrix of $z$. Explicitly, $z^{*}$ (as an element of $\mathbb{C}\left(\mathbb{C}^{2}\right)$ ) is given by 


$$
\begin{aligned}
z^{*}= & \overline{\left(z^{t}\right)} \\
= & x_{0}-x_{1} \varepsilon_{1}+x_{2} \varepsilon_{2}-x_{3} \varepsilon_{3}+x_{4} \varepsilon_{4}-x_{5} \varepsilon_{5}-x_{6} \varepsilon_{6}+x_{7} \varepsilon_{7} \\
= & \left\{\left(x_{0}-x_{1} \varepsilon_{1}\right)+\left(x_{2}-x_{3} \varepsilon_{1}\right) \varepsilon_{2}\right\} \\
& +\left\{\left(x_{4}-x_{5} \varepsilon_{1}\right)-\left(x_{6}-x_{7} \varepsilon_{1}\right) \varepsilon_{2}\right\} \varepsilon_{4} \\
= & \left(\bar{\zeta}_{0}+\bar{\zeta}_{1} \varepsilon_{2}\right)+\left(\bar{\zeta}_{2}-\bar{\zeta}_{3} \varepsilon_{2}\right) \varepsilon_{4} \\
= & \bar{z}_{0}+\overline{\bar{z}}_{1} \varepsilon_{4} .
\end{aligned}
$$

From the above results, the norm $\|z\|$ of $z$ is therefore given by

$$
\|z\|=\frac{1}{2}\left\{\operatorname{tr}\left(z z^{*}\right)\right\}^{1 / 2}=\left(\sum_{j=0}^{7} x_{j}^{2}\right)^{1 / 2}
$$

where $\operatorname{tr}(z)$ is the trace of matrix $\left(z z^{*}\right)$.

Proposition 1. For $z, w \in \mathbb{C}\left(\mathbb{C}^{2}\right)$, the following properties for the norm $\|z\|$ are satisfied:

(1) $\|z+w\| \leq\|z\|+\|w\|$

(2) $\|z w\| \leq\|z\|\|w\|, \quad z, w \in \mathbb{C}\left(\mathbb{C}^{2}\right)$

We now define differential operators as algebraic expressions for the elemental form of $\mathbb{C}\left(\mathbb{C}^{2}\right)$. The differential operator here includes elements in the form of a matrix. We consider the following differential operators over $\mathbb{C}\left(\mathbb{C}^{2}\right)$ :

$$
\begin{aligned}
& \widetilde{D}_{1}=\frac{1}{2}\left(\frac{\partial}{\partial \widetilde{z}_{0}}-\varepsilon_{4} \frac{\partial}{\partial z_{1}}\right), \\
& D_{1}=\frac{1}{2}\left(\frac{\partial}{\partial z_{0}}+\varepsilon_{4} \frac{\partial}{\partial z_{1}}\right) \text {, } \\
& \widetilde{D}_{2}=\frac{1}{2}\left(\frac{\partial}{\partial z_{0}}-\varepsilon_{4} \frac{\partial}{\partial z_{1}}\right) \text {, } \\
& D_{2}=\frac{1}{2}\left(\frac{\partial}{\partial \widetilde{z}_{0}}+\varepsilon_{4} \frac{\partial}{\partial z_{1}}\right) \text {, } \\
& \widetilde{D}_{3}=\frac{1}{2}\left(\frac{\partial}{\partial \widetilde{z}_{0}}-\varepsilon_{4} \frac{\partial}{\partial \widetilde{z}_{1}}\right), \\
& D_{3}=\frac{1}{2}\left(\frac{\partial}{\partial z_{0}}+\varepsilon_{4} \frac{\partial}{\partial \widetilde{z}_{1}}\right) \text {, } \\
& \widetilde{D}_{4}=\frac{1}{2}\left(\frac{\partial}{\partial z_{0}}-\varepsilon_{4} \frac{\partial}{\partial \widetilde{z}_{1}}\right) \text {, } \\
& D_{4}=\frac{1}{2}\left(\frac{\partial}{\partial \widetilde{z}_{0}}+\varepsilon_{4} \frac{\partial}{\partial \widetilde{z}_{1}}\right), \\
& \frac{\partial}{\partial z_{0}}=\frac{1}{2}\left(\frac{\partial}{\partial \zeta_{0}}+\varepsilon_{2} \frac{\partial}{\partial \zeta_{1}}\right), \\
& \frac{\partial}{\partial z_{1}}=\frac{1}{2}\left(\frac{\partial}{\partial \zeta_{2}}+\varepsilon_{2} \frac{\partial}{\partial \zeta_{3}}\right), \\
& \frac{\partial}{\partial \widetilde{z}_{0}}=\frac{1}{2}\left(\frac{\partial}{\partial \zeta_{0}}-\varepsilon_{2} \frac{\partial}{\partial \zeta_{1}}\right), \\
& \frac{\partial}{\partial \widetilde{z}_{1}}=\frac{1}{2}\left(\frac{\partial}{\partial \zeta_{2}}-\varepsilon_{2} \frac{\partial}{\partial \zeta_{3}}\right),
\end{aligned}
$$


where $\partial / \partial \zeta_{j} \quad(j=0,1,2,3)$ has the same pattern operator form as usual complex differential operators.

As a concrete example, we have

$$
\begin{aligned}
& \frac{\partial}{\partial \zeta_{0}} \zeta_{0}=\frac{1}{2}\left(\frac{\partial}{\partial x_{0}}-\varepsilon_{1} \frac{\partial}{\partial x_{1}}\right)\left(x_{0}+x_{1} \varepsilon_{1}\right) \\
& =\frac{1}{2}\left(\begin{array}{cccc}
\frac{\partial}{\partial x_{0}}-i \frac{\partial}{\partial x_{1}} & 0 & 0 & 0 \\
0 & \frac{\partial}{\partial x_{0}}+i \frac{\partial}{\partial x_{1}} & 0 & 0 \\
0 & 0 & \frac{\partial}{\partial x_{0}}+i \frac{\partial}{\partial x_{1}} & 0 \\
0 & 0 & 0 & \frac{\partial}{\partial x_{0}}-i \frac{\partial}{\partial x_{1}}
\end{array}\right) \\
& \left(\begin{array}{cccc}
x_{0}+i x_{1} & 0 & 0 & 0 \\
0 & x_{0}-i x_{1} & 0 & 0 \\
0 & 0 & x_{0}-i x_{1} & 0 \\
0 & 0 & 0 & x_{0}+i x_{1}
\end{array}\right) \\
& =\left(\begin{array}{llll}
1 & 0 & 0 & 0 \\
0 & 1 & 0 & 0 \\
0 & 0 & 1 & 0 \\
0 & 0 & 0 & 1
\end{array}\right) .
\end{aligned}
$$

In this case, let the $4 \times 4$ identity matrix be 1 . If the calculation is performed by expressing it as a matrix, the following results are obtained:

$$
\begin{gathered}
\frac{\partial}{\partial z_{0}} z_{0}=1, \\
\frac{\partial}{\partial \widetilde{z}_{0}} z_{0}=0 .
\end{gathered}
$$

Next, let $\Omega$ be a domain in $\mathbb{C}^{4}$. We consider a function $f: \Omega \longrightarrow \mathbb{C}\left(\mathbb{C}^{2}\right)$ by

$$
\begin{aligned}
z & =\left(z_{0}, z_{1}\right) \mapsto f(z) \\
& =f_{0}\left(z_{0}, z_{1}\right)+f_{1}\left(z_{0}, z_{1}\right) \varepsilon_{4},
\end{aligned}
$$

where $f_{0}$ and $f_{1}$ have the form $\varphi+\psi \varepsilon_{2}$ with $\varphi$ and $\psi$ being complex-valued functions. For example, for a function $f(z)=z^{2}$, this takes the form $f(z)=\left(z_{0}^{2}+z_{1} \widetilde{z}_{1}\right)$ $+\left(z_{0} z_{1}+z_{1} \widetilde{z}_{0}\right) \varepsilon_{4}$, denoted by $f_{0}=z_{0}^{2}+z_{1} \widetilde{z}_{1}$ and $f_{1}=z_{0} z_{1}+z_{1} \tilde{z}_{0}$.
The differential operator $\widetilde{D}_{k}(k=1,2,3,4)$ operates on $f$ as follows: in the case of $n=1$, applying the differential operator from the left, we have

$$
\begin{aligned}
\widetilde{D}_{1} f & =\frac{1}{2}\left(\frac{\partial}{\partial \widetilde{z}_{0}}-\varepsilon_{4} \frac{\partial}{\partial z_{1}}\right)\left(f_{0}+f_{1} \varepsilon_{4}\right) \\
& =\frac{1}{2}\left\{\left(\frac{\partial f_{0}}{\partial \widetilde{z}_{0}}-\frac{\partial \widetilde{f}_{1}}{\partial \widetilde{z}_{1}}\right)+\left(\frac{\partial f_{1}}{\partial \widetilde{z}_{0}}-\frac{\partial \widetilde{f}_{0}}{\partial \widetilde{z}_{1}}\right) \varepsilon_{4}\right\},
\end{aligned}
$$

and applying the differential operator from the right, we obtain

$$
\begin{aligned}
f \widetilde{D}_{1} & =\frac{1}{2}\left(f_{0}+f_{1} \varepsilon_{4}\right)\left(\frac{\partial}{\partial \widetilde{z}_{0}}-\varepsilon_{4} \frac{\partial}{\partial z_{1}}\right) \\
& =\frac{1}{2}\left\{\left(\frac{\partial f_{0}}{\partial \widetilde{z}_{0}}-\frac{\partial f_{1}}{\partial z_{1}}\right)+\left(-\frac{\partial f_{0}}{\partial \widetilde{z}_{1}}+\frac{\partial f_{1}}{\partial z_{0}}\right) \varepsilon_{4}\right\} .
\end{aligned}
$$

Now, let us define the regular functions to be applied in $\mathbb{C}\left(\mathbb{C}^{2}\right)$ by using the differential operator $\widetilde{D}_{k}\left(D_{k}\right)(k=1,2,3,4)$ for functions defined in $\mathbb{C}\left(\mathbb{C}^{2}\right)$.

\section{Definitions and Properties of Regularity}

Definition 1. Let $\Omega$ be a domain in $\mathbb{C}^{4}$. Let $f=f_{0}+f_{1} \varepsilon_{4}$ be a function defined on $\Omega$. The function $f$ is said to be $J_{L_{k}}\left(J_{R_{k}}\right)$-regular function $(k=1,2,3,4)$ in $\Omega$ if

(1) $f_{0}$ and $f_{1}$ are separately continuously holomorphic functions defined on $\Omega$

(2) $\widetilde{D}_{k} f=0, \quad\left(f \widetilde{D}_{k}=0\right)$ in $\Omega$, where $k=1,2,3,4$

When a function $f$ is $J_{L_{k}}\left(J_{R_{k}}\right)$-regular $(k=1,2,3,4)$, it means that at least the above definition is satisfied for at least one of $k=1,2,3,4$.

Remark 1. Regarding $f_{0}$ and $f_{1}$ being separately continuous holomorphic functions, we mean that $f_{0}=\varphi_{1}+\varphi_{2} \varepsilon_{2}$ and $f_{1}=\psi_{1}+\psi_{2} \varepsilon_{2}$ such that the components $\varphi_{r}$ and $\psi_{r}(r=$ $1,2)$ of each $f_{t}(t=0,1)$ are holomorphic complex-valued functions.

Lemma 1 (Cauchy-Riemann equation in $\mathbb{C}\left(\mathbb{C}^{2}\right)$ ). Condition 2 of Definition 1 for $J_{L_{k}}$-regular function is equivalent to

$$
\begin{aligned}
& \frac{\partial f_{0}}{\partial \widetilde{z}_{0}}=\frac{\partial \tilde{f}_{1}}{\partial \widetilde{z}_{1}} \frac{\partial f_{1}}{\partial \widetilde{z}_{0}}=\frac{\partial \tilde{f}_{0}}{\partial \widetilde{z}_{1}}, \quad \text { if } k=1, \\
& \frac{\partial f_{0}}{\partial z_{0}}=\frac{\partial \tilde{f}_{1}}{\partial \widetilde{z}_{1}}, \frac{\partial f_{1}}{\partial z_{0}}=\frac{\partial \tilde{f}_{0}}{\partial \widetilde{z}_{1}}, \quad \text { if } k=2, \\
& \frac{\partial f_{0}}{\partial \widetilde{z}_{0}}=\frac{\partial \tilde{f}_{1}}{\partial z_{1}}, \frac{\partial f_{1}}{\partial \widetilde{z}_{0}}=\frac{\partial \tilde{f}_{0}}{\partial z_{1}}, \quad \text { if } k=3, \\
& \frac{\partial f_{0}}{\partial z_{0}}=\frac{\partial \tilde{f}_{1}}{\partial z_{1}}, \frac{\partial f_{1}}{\partial z_{0}}=\frac{\partial \tilde{f}_{0}}{\partial z_{1}}, \quad \text { if } k=4 .
\end{aligned}
$$


In addition, for $J_{R_{k}}$-regular function, Condition 2 is equivalent to

$$
\begin{array}{ll}
\frac{\partial f_{0}}{\partial \widetilde{z}_{0}}=\frac{\partial f_{1}}{\partial z_{1}}, \frac{\partial f_{1}}{\partial z_{0}}=\frac{\partial f_{0}}{\partial \widetilde{z}_{1}}, & \text { if } k=1, \\
\frac{\partial f_{0}}{\partial z_{0}}=\frac{\partial f_{1}}{\partial z_{1}}, \frac{\partial f_{1}}{\partial \widetilde{z}_{0}}=\frac{\partial \widetilde{f}_{0}}{\partial \widetilde{z}_{1}}, & \text { if } k=2, \\
\frac{\partial f_{0}}{\partial \widetilde{z}_{0}}=\frac{\partial f_{1}}{\partial \widetilde{z}_{1}}, \frac{\partial f_{1}}{\partial z_{0}}=\frac{\partial f_{0}}{\partial z_{1}}, & \text { if } k=3, \\
\frac{\partial f_{0}}{\partial z_{0}}=\frac{\partial f_{1}}{\partial \widetilde{z}_{1}}, \frac{\partial f_{1}}{\partial \widetilde{z}_{0}}=\frac{\partial f_{0}}{\partial z_{1}}, & \text { if } k=4 .
\end{array}
$$

Theorem 1. Let $\Omega$ be an open set in $\mathbb{C}^{4}$, and let $f$ be a regular function on $\Omega$. Then, the derivative $f^{\prime}$ of $f$, also denoted as $D f$, is defined as

$$
f^{\prime}=\frac{\partial f}{\partial x_{0}}=-\widehat{i} \frac{\partial f}{\partial z_{0}}
$$

on $\Omega$.

Proof. From the definition of $J_{L_{k}}$-regular function defined on $\mathbb{C}\left(\mathbb{C}^{2}\right)$, in case $k=1$, Condition 2 can be expressed as follows:

$$
\widetilde{D}_{1} f=\frac{1}{2}\left\{\left(\frac{\partial f_{0}}{\partial \widetilde{z}_{0}}-\frac{\partial \widetilde{f}_{1}}{\partial \widetilde{z}_{1}}\right)+\left(\frac{\partial f_{1}}{\partial \widetilde{z}_{0}}-\frac{\partial \widetilde{f}_{0}}{\partial \widetilde{z}_{1}}\right) \varepsilon_{4}\right\}
$$

Since $f$ satisfies

$$
=0 \text {. }
$$

$$
\begin{aligned}
& \frac{\partial f_{0}}{\partial \widetilde{z}_{0}}-\frac{\partial \tilde{f}_{1}}{\partial \widetilde{z}_{1}}=0, \\
& \frac{\partial f_{1}}{\partial \widetilde{z}_{0}}-\frac{\partial \tilde{f}_{0}}{\partial \widetilde{z}_{1}}=0,
\end{aligned}
$$

we obtain

$$
\begin{aligned}
& \frac{\partial f_{0}}{\partial \widetilde{z}_{0}}=\frac{\partial \tilde{f}_{1}}{\partial \widetilde{z}_{1}}, \\
& \frac{\partial f_{1}}{\partial \widetilde{z}_{0}}=\frac{\partial \widetilde{f}_{0}}{\partial \widetilde{z}_{1}} .
\end{aligned}
$$

Similarly, we can derive the Cauchy-Riemann equations for the other cases.

For example, let $f$ be an identity function such that $f(z)=z$. The function $f$ is $J_{L_{k}}$-regular $(k=1,2,3,4)$ defined on $\mathbb{C}\left(\mathbb{C}^{2}\right)$. Let $f$ be a function such that $f(z)=z^{2}$ be $J_{L_{1}}$-regular defined on $\mathbb{C}\left(\mathbb{C}^{2}\right)$. Let $f$ be $J_{L_{k}}\left(J_{R_{k}}\right)$-regular functions $(k=1,2,3,4)$ defined in $\Omega$. Then, we propose the $J_{L_{k}}\left(J_{R_{k}}\right)$-derivative $D_{k} f\left(f D_{k}\right)(k=1,2,3,4)$ of $f$ and find the following theorem.
Theorem 2. Let $\Omega$ be a domain in $\mathbb{C}^{4}$. Let $f$ be a $J_{L_{k}}\left(J_{R_{k}}\right)$-regular function defined in $\Omega$. Then, the derivative $D_{k} f\left(f D_{k}\right)(k=1,2,3,4)$ of $f$ satisfies

$$
D_{k} f=f D_{k}=\frac{\partial f}{\partial \zeta_{0}}, \quad(k=1,2,3,4) .
$$

Proof. Since $f$ is $J_{L_{1}}$-regular defined on $\Omega$, the function $f$ satisfies $\widetilde{D}_{1} f=0$. Hence, we have

$$
\begin{aligned}
D_{1} f & =\frac{1}{2}\left\{\left(\frac{\partial f_{0}}{\partial z_{0}}+\frac{\partial \widetilde{f}_{1}}{\partial \widetilde{z}_{1}}\right)+\left(\frac{\partial f_{1}}{\partial z_{0}}-\frac{\partial \widetilde{f}_{0}}{\partial \widetilde{z}_{1}}\right) \varepsilon_{4}\right\} \\
& =\frac{1}{2}\left\{\left(\frac{\partial f_{0}}{\partial z_{0}}+\frac{\partial f_{0}}{\partial \widetilde{z}_{0}}\right)+\left(\frac{\partial f_{1}}{\partial z_{0}}+\frac{\partial f_{1}}{\partial \widetilde{z}_{0}}\right) \varepsilon_{4}\right\} \\
& =\frac{1}{2}\left(\frac{\partial f}{\partial z_{0}}+\frac{\partial f}{\partial \widetilde{z}_{0}}\right) \\
& =\frac{\partial f}{\partial \zeta_{0}} .
\end{aligned}
$$

When $k=2,3,4$, by using the equation $\widetilde{D}_{k} f=0$, we obtain the same results, that is,

$$
D_{2} f=D_{3} f=D_{4} f=\frac{1}{2}\left(\frac{\partial f}{\partial z_{0}}+\frac{\partial f}{\partial \widetilde{z}_{0}}\right)=\frac{\partial f}{\partial \zeta_{0}} .
$$

Similarly, when $f$ is $J_{R_{k}}$-regular $(k=1,2,3,4)$ defined on $\Omega$, then by the equation $f \widetilde{D}_{k}=0(k=1,2,3,4)$, we obtain

$$
f D_{k}=\frac{1}{2}\left(\frac{\partial f}{\partial z_{0}}+\frac{\partial f}{\partial \widetilde{z}_{0}}\right), \quad(k=1,2,3,4) .
$$

Thus, the $J_{L_{k}}\left(J_{R_{k}}\right)$-derivative of $f$ satisfies

$$
D_{k} f=f D_{k}=\frac{\partial f}{\partial \zeta_{0}}, \quad(k=1,2,3,4) .
$$

From Theorem 1, for a separately continuous holomorphic $J_{L_{k}}\left(J_{R_{k}}\right)$-regular function $f(k=1,2,3,4)$ defined in $\Omega$, we denote

$$
\left(D_{k}^{n} f\right)(z)=\left(f D_{k}^{n}\right)(z)=\frac{\partial^{n}}{\partial \zeta_{0}^{n}} f(z)=f(z) \frac{\partial^{n}}{\partial \zeta_{0}^{n}} .
$$

\section{Properties of $J_{L_{k}}\left(J_{R_{k}}\right)$-Regular}

\section{$(k=1,2,3,4)$ Functions}

In this section, let $\Omega$ be a domain in $\mathbb{C}^{4}$. Based on the definition and properties of $J_{L_{k}}$-regularity $(k=1,2,3,4)$, we determine whether the properties of holomorphic function of complex variables extend $J_{L_{k}}$-regular function $(k=1,2,3,4)$ defined on $\Omega$. Suppose that $\Omega_{1}, \Omega_{2} \subset \mathbb{C}^{4}$ are two open neighborhoods; then, the variables in $\Omega_{1}$ can be written as $z=\left(z_{0}, z_{1}\right)$ and $\Omega_{2}$ can be written as $w=\left(w_{0}, w_{1}\right)$. Any mapping $g: \Omega_{1} \longrightarrow \Omega_{2}$ can be described by 


$$
\begin{aligned}
& w_{0}=g_{0}\left(z_{0}, z_{1}\right), \\
& w_{1}=g_{1}\left(z_{0}, z_{1}\right) .
\end{aligned}
$$

The mapping $g$ is called a $J_{L_{k}}$-regular mapping $(k=$ $1,2,3,4)$ if the functions $g_{0}$ and $g_{1}$ are $J_{L_{k}}$-regular functions $(k=1,2,3,4)$ in $\Omega_{1}$. If $f\left(w_{0}, w_{1}\right)=f(w)$ is any function defined in $\Omega_{2}$, then composition $f(g)$ is a well-defined function in $\Omega_{1}$. If $f(w)$ is a $J_{L_{k}}$-regular function $(k=1,2,3,4)$ in $\Omega_{2}$ and if $g: \Omega_{1} \longrightarrow \Omega_{2}$ is a $J_{L_{k}}$-regular mapping $(k=1,2,3,4)$, then the composition $w=f(g)$ is a $J_{L_{k}}$-regular function $(k=1,2,3,4)$ in $\Omega_{1}$.

Next, the following quadratic form is factors that play an important role in the integral calculus of $J_{L_{k}}$-regular functions $(k=1,2,3,4)$ :

$$
\begin{aligned}
& \omega_{1}=\mathrm{d} z_{0} \wedge \mathrm{d} z_{1} \wedge \mathrm{d} \widetilde{z}_{1}-\mathrm{d} z_{0} \wedge \mathrm{d} \widetilde{z}_{0} \wedge \mathrm{d} z_{1} \varepsilon_{4}, \\
& \omega_{2}=\mathrm{d} \widetilde{z}_{0} \wedge \mathrm{d} z_{1} \wedge \mathrm{d} \bar{z}_{1}+\mathrm{d} z_{0} \wedge \mathrm{d} \widetilde{z}_{0} \wedge \mathrm{d} z_{1} \varepsilon_{4}, \\
& \omega_{3}=\mathrm{d} z_{0} \wedge \mathrm{d} z_{1} \wedge \mathrm{d} \widetilde{z}_{1}+\mathrm{d} z_{0} \wedge \mathrm{d} \widetilde{z}_{0} \wedge \mathrm{d} \widetilde{z}_{1} \varepsilon_{4}, \\
& \omega_{4}=\mathrm{d} \widetilde{z}_{0} \wedge \mathrm{d} z_{1} \wedge \mathrm{d} \widetilde{z}_{1}-\mathrm{d} z_{0} \wedge \mathrm{d} \widetilde{z}_{0} \wedge \mathrm{d} \widetilde{z}_{1} \varepsilon_{4} .
\end{aligned}
$$

Furthermore, we note that

$$
\begin{aligned}
\mathrm{d} z_{i} \wedge \mathrm{d} z_{j} & =-\mathrm{d} z_{j} \wedge \mathrm{d} z_{i}, \\
\mathrm{~d} z_{i} \wedge \mathrm{d} z_{i} & =0, \quad(i, j=0,1), \\
\mathrm{d} \Phi: & =\mathrm{d} z_{0} \wedge \mathrm{d} \widetilde{z}_{0} \wedge \mathrm{d} z_{1} \wedge \mathrm{d} \widetilde{z}_{1} .
\end{aligned}
$$

Definition 2 (Morera's theorem in $\mathbb{C}\left(\mathbb{C}^{2}\right)$ ). Let a function $f$ be continuous in $\Omega$. Suppose the integral of $\omega_{k} f(k=1,2,3,4)$ over the boundary $\partial U$ of any sphere $U$ in $\Omega$ be equal to zero. Then, $f$ is a $J_{L_{k}}$-regular function $(k=$ $1,2,3,4)$ in $\Omega$.

Proof. We assume that the function $f$ is smooth in $\Omega$. Let $U$ be a sphere in $\Omega$. Based on this hypothesis, we have

$$
\int_{\partial U} \omega_{k} f=0, \quad(k=1,2,3,4) .
$$

From Stokes' formula in complex analysis, we obtain

$$
\int_{U} d_{k}\left(\omega_{k} f\right)=\int_{\partial U} \omega_{k} f=0, \quad(k=1,2,3,4) .
$$

Hence, for $k=1$, the integral can be calculated as follows:

$$
\int_{U}\left(\frac{\partial f_{0}}{\partial \widetilde{z}_{0}}-\frac{\partial \widetilde{f}_{1}}{\partial \widetilde{z}_{1}}\right) \mathrm{d} \Phi=\int_{U}\left(\frac{\partial f_{1}}{\partial \widetilde{z}_{0}}+\frac{\partial \widetilde{f}_{0}}{\partial \widetilde{z}_{1}}\right) \mathrm{d} \Phi=0 .
$$

Due to the smoothness of $f$, the expression under the integral sign is continuous. Since the fact $U$ is an arbitrary sphere in $\Omega$, it follows that

$$
\begin{aligned}
& \frac{\partial f_{0}}{\partial \widetilde{z}_{0}}=\frac{\partial \tilde{f}_{1}}{\partial \widetilde{z}_{1}} \\
& \frac{\partial f_{1}}{\partial \widetilde{z}_{0}}=-\frac{\partial \tilde{f}_{0}}{\partial \widetilde{z}_{1}},
\end{aligned}
$$

in $\Omega$. For cases $k=2,3,4$, we can obtain similar conclusions.
Next, we consider the relationship between the $J_{L_{k}}$-regular function $(k=1,2,3,4)$ and series expansions in $\mathbb{C}\left(\mathbb{C}^{2}\right)$.

Lemma 2. Let $f$ be a homogeneous polynomial of degree $n$ with respect to $z_{0}$ and $z_{1}$. If $f$ is a $J_{L_{k}}$-regular function ( $k=$ $1,2,3,4)$ in $\mathbb{C}^{4}$, then $f$ satisfies

$$
f(z)=\frac{1}{n !} \frac{\partial^{n} f(z)}{\partial z_{0}^{n}} z^{n} .
$$

Proof. According to the hypothesis, the function $f(z)$ is a homogeneous polynomial; thus, $f$ satisfies

$$
f(z)=\frac{1}{n} \frac{\partial f(z)}{\partial z_{0}} z
$$

Since $\partial f / \partial z_{0}$ is a homogeneous polynomial of degree $n-1$, we have

$$
\frac{\partial f(z)}{\partial z_{0}}=\frac{1}{n-1} \frac{\partial^{2} f(z)}{\partial z_{0}^{2}} z
$$

Continuing this process, we can obtain

$$
f(z)=\frac{1}{n !} \frac{\partial^{n} f(z)}{\partial z_{0}^{n}} z^{n} .
$$

Proposition 2. Let $\Omega$ be a domain in $\mathbb{C}^{4}$ and $f(z)$ be a function defined on a neighborhood $V$ of $\alpha \in \mathbb{C}^{4}$ with values in $\mathbb{C}\left(\mathbb{C}^{2}\right)$. The function $f$ is a $J_{L_{k}}$-regular function $(k=1,2,3,4)$ in $\Omega$ and $\alpha \in \Omega$ if and only if $f$ has a power series expansion in $V$ such that for $z \in V$,

$$
f(z)=\sum_{n=0}^{\infty} a_{n}(z-\alpha)^{n}
$$

where $a_{n}=(1 / n !)\left(\partial^{n} f(\alpha) / \partial z_{0}^{n}\right)(n=0,1,2, \ldots)$.

Proof. Without loss of generality, let $\alpha=0$. Suppose that $f$ is $J_{L_{k}}$-regular $(k=1,2,3,4)$ in $\Omega$ with a value in $\mathbb{C}\left(\mathbb{C}^{2}\right)$; then, $f$ is holomorphic in $\Omega$. Thus, there exists a neighborhood $V$ of zero such that for $z \in V$,

$$
f(z)=\sum_{n=0}^{\infty} P_{n}(z)
$$

where $P_{n}(z)$ are homogeneous polynomials of degree $n$ with respect to $z_{0}$ and $z_{1}$. Since the above series converges uniformly in $V$ and $P_{n}(z)$ are homogeneous polynomials of degree $n$ with respect to $z_{0}$ and $z_{1}$, when $k=1$, we have

$$
\begin{aligned}
D_{1}^{*} f(z) & =\frac{1}{2}\left(\frac{\partial}{\partial \bar{z}_{0}}+\varepsilon_{4} \frac{\partial}{\partial z_{1}}\right) f(z) \\
& =\sum_{n=0}^{\infty} \frac{1}{2}\left(\frac{\partial}{\partial \bar{z}_{0}}+\varepsilon_{4} \frac{\partial}{\partial z_{1}}\right) P_{n}(z)=0 .
\end{aligned}
$$

Hence, $P_{n}(z)$ is a $J_{L_{k}}$-regular $(k=1,2,3,4)$ in $\Omega$. By Lemma 2, since 


$$
\frac{\partial^{n} f(0)}{\partial z_{0}^{n}}=\frac{\partial^{n}}{\partial z_{0}^{n}} \sum_{n=0}^{\infty} P_{n}(z)
$$

we have

$$
\begin{aligned}
f(z) & =\sum_{n=0}^{\infty} P_{n}(z) \\
& =\sum_{n=0}^{\infty} \frac{1}{n !} \frac{\partial^{n} P_{n}(z)}{\partial z_{0}^{n}} z^{n} \\
& =\sum_{n=0}^{\infty} \frac{1}{n !} \frac{\partial^{n} f(0)}{\partial z_{0}^{n}} z^{n} \\
& =\sum_{n=0}^{\infty} a_{n} z^{n}, \\
z & \in V .
\end{aligned}
$$

Suppose that

$$
f(z)=\sum_{n=0}^{\infty} a_{n} z^{n}
$$

where $a_{n}=(1 / n !)\left(\partial^{n} f(0) / \partial z_{0}^{n}\right) \quad(n=0,1,2, \ldots)$. Since $f(z)$ converges uniformly in $V$, we obtain

$$
\begin{aligned}
D_{1}^{*} f(z) & =\frac{1}{2}\left(\frac{\partial}{\partial \bar{z}_{0}}+\varepsilon_{4} \frac{\partial}{\partial z_{1}}\right) f(z) \\
& =\sum_{n=0}^{\infty} \frac{1}{2} a_{n}\left(\frac{\partial}{\partial \bar{z}_{0}}+\varepsilon_{4} \frac{\partial}{\partial z_{1}}\right) z^{n} \\
& =\frac{1}{2} \sum_{n=0}^{\infty} a_{n}\left(\frac{\partial}{\partial \bar{z}_{0}}\left(z_{0}+z_{1} \varepsilon_{4}\right)^{n}+\varepsilon_{4} \frac{\partial}{\partial z_{1}}\left(z_{0}+z_{1} \varepsilon_{4}\right)^{n}\right) \\
& =\frac{1}{2} \sum_{n=0}^{\infty} a_{n}\left(n\left(z_{0}+\varepsilon_{2} z_{1}\right)^{n-1}+\varepsilon_{2} n\left(z_{0}+\varepsilon_{2} z_{1}\right)^{n-1} \varepsilon_{2}\right) \\
& =0 .
\end{aligned}
$$

Thus, $f(z)$ is a $J_{L_{k}}$-regular function $(k=1,2,3,4)$ in $V$.

From Proposition 2, equivalently, we can define $J_{L_{k}}$-regular functions $(k=1,2,3,4)$ in $\Omega$ which is a domain in $\mathbb{C}^{4}$ as follows.

Definition 3. Let $\Omega$ be a domain in $\mathbb{C}^{4}$. A function $f=$ $f_{0}+f_{1} \varepsilon_{4}$ is said to a $J_{L_{k}}$-regular function $(k=1,2,3,4)$ on $\Omega$ if every point $a \in \Omega$ corresponds to a neighborhood $U$ admitting a power series

$$
\sum_{n=0}^{\infty} a_{n}(z-\alpha)^{n}
$$

which converges to $f(z)$ for $z \in U$.

From the above results, the following statements are equivalent to each other:
(1) $f$ is a $J_{L_{k}}$-regular function $(k=1,2,3,4)$ defined on $\Omega$.

(2) For every point $a \in \Omega$, there is a neighborhood $U$ admitting a power series

$$
f(z)=\sum_{n=0}^{\infty} a_{n}(z-\alpha)^{n}, \quad z \in U .
$$

(3) For a smooth boundary $\partial U$ such that $\widetilde{U} \subset \Omega$, we have

$$
\int_{\partial U} \omega_{k} f=0
$$

where $\omega_{k} f(k=1,2,3,4)$ is the quaternion product of the form of the function $f=f_{1}+f_{2} \varepsilon_{4}$.

Corollary 1. Let $\Omega$ be a domain in $\mathbb{C}^{4}$ and $f$ be a $J_{L_{k}}$-regular function $(k=1,2,3,4)$. If $f$ is infinitely differentiable in $\mathbb{C}\left(\mathbb{C}^{2}\right)$ and

$$
f(z)=\sum_{n=0}^{\infty} a_{n}(z-\alpha)^{n}
$$

then we have

$$
a_{n}=\frac{1}{n !}\left(D_{k}^{n} f\right)(\alpha)=\frac{1}{n !} \frac{\partial^{n}}{\partial z_{0}^{n}} f(\alpha), \quad n=0,1,2, \ldots
$$

\section{Conclusion}

In this paper, we developed the foundation of a function theory over a subalgebra of four-dimensional matrix algebra as an alternative to the standard basis of quaternion algebra. Furthermore, we introduced a semicommutative subalgebra $\mathbb{C}\left(\mathbb{C}^{2}\right)$ of the complex matrix algebra $M(4, \mathbb{C})$, and we propose regular functions defined in a domain in $\mathbb{C}^{4}$ with values in $\mathbb{C}\left(\mathbb{C}^{2}\right)$. From the definition of regularity over $\mathbb{C}\left(\mathbb{C}^{2}\right)$, we derived corresponding Cauchy-Riemann equations and several properties of regular functions in $\mathbb{C}\left(\mathbb{C}^{2}\right)$. Throughout this paper, it has been possible to make various attempts to utilize quaternions by demonstrating a series expansion of quaternion variable. Regular functions defined on quaternions can be applied to this extension of the algebra of quaternions introduced in this paper. In addition, it is possible to present the extensibility of formulas derived in this paper based on proposed quaternionic theory for differential operators and series expansion. In the future, we plan to verify properties, such as $\bar{\partial}$-closed form and regularity over a domain of holomorphy, by using certain regular functions and Cauchy-Riemann equations.

\section{Data Availability}

No datasets were generated or analyzed during the current study.

\section{Conflicts of Interest}

The author declares no conflicts of interest. 


\section{Acknowledgments}

This study was supported by the Dongguk University Research Fund 2020 and the National Research Foundation of Korea (NRF) (2021R1F1A1063356).

\section{References}

[1] M. Geradin and A. Cardona, "Kinematics and dynamics of rigid and flexible mechanisms using finite elements and quaternion algebra," Computational Mechanics, vol. 4, no. 2, pp. 115-135, 1988.

[2] R. E. Spall and W. Yu, "Imbedded dual-number automatic differentiation for computational fluid dynamics sensitivity analysis," Journal of Fluids Engineering, vol. 135, no. 1, 2013.

[3] J. Turner, "Quaternion-based partial derivative and state transition matrix calculations for design optimization," in Proceedings of the 40th AIAA Aerospace Sciences Meeting \& Exhibit, p. 448, Reno, Nevada, January 2002.

[4] R. Fueter, "Die Funktionentheorie der Differentialgleichungen? $\mathrm{u}=0$ und? $\mathrm{u}=0$ mit vier reellen Variablen," Commentarii Mathematici Helvetici, vol. 7, no. 1, pp. 307-330, 1934.

[5] R. Delanghe, "On regular-analytic functions with values in a Clifford algebra," Mathematische Annalen, vol. 185, no. 2, pp. 91-111, 1970.

[6] J. Ryan, "Complexified Clifford analysis," Complex Variables, Theory and Application, vol. 1, no. 1, pp. 119-149, 1983.

[7] A. Sudbery, "Quaternionic analysis," Mathematical Proceedings of the Cambridge Philosophical Society, vol. 85, no. 2, pp. 199-225, 1979.

[8] M. Naser, "Hyperholomorphic functions," Siberian Mathematical Journal, vol. 12, pp. 959-968, 1971.

[9] K. Nôno, "Hyperholomorphic functions of a quaternion variable," Bulletin of Fukuoka University of Education, vol. 32, pp. 21-37, 1982.

[10] K. Nôno and Y. Inenaga, "On quaternionic analysis of holomorphic mappings in $\mathbb{C}^{2}$," Bulletin of Fukuoka University of Education, vol. 37, pp. 17-27, 1988.

[11] J. E. Kim, S. J. Lim, and K. H. Shon, "Regular functions with values in ternary number system on the complex Clifford analysis," Abstract and Applied Analysis, vol. 2013, Article ID 136120, 7 pages, 2013.

[12] J. E. Kim, S. J. Lim, and K. H. Shon, "Regularity of functions on the reduced quaternion field in Clifford analysis," Abstract and Applied Analysis, vol. 2014, Article ID 654798, 8 pages, 2014.

[13] F. Sommen, "Some connections between Clifford analysis and complex analysis," Complex Variables, Theory and Application: An International Journal, vol. 1, no. 1, pp. 97-118, 1982.

[14] J. Baez, "The octonions," Bulletin of the American Mathematical Society, vol. 39, no. 2, pp. 145-205, 2002.

[15] K. Imaeda and M. Imaeda, "Sedenions: algebra and analysis," Applied Mathematics and Computation, vol. 115, no. 2-3, pp. 77-88, 2000.

[16] Y. Tian, "Matrix representations of octonions and their applications," Advances in Applied Clifford Algebras, vol. 10, no. 1, pp. 61-90, 2000.

[17] S. Gotô and K. Nôno, "Regular functions with values in a commutative subalgebra $\mathbb{C}(\mathbb{C})$ of matrix algebra $M(4, \mathbb{R})$," Bulletin of Fukuoka University of Education, vol. 61, pp. 9-15, 2012.

[18] W. Rudin, Function Theory in the Unit Ball of $\mathbb{C}^{n}$, Vol. 241, Springer Science \& Business Media, , New York, NY, USA, 1980. 